\title{
A REMARK CONCERNING A MECHANICAL CHARACTERIZATION OF THE SPHERE
}

\author{
PARIS PAMFILOS
}

\begin{abstract}
It is shown that if a (sufficiently) small ball rolls freely without sliding on a compact surface in such a way that its center moves with constant in magnitude velocity for every initial condition, then the surface must be a sphere.
\end{abstract}

There are many interesting problems relating the mechanics of a ball rolling (without sliding) freely on a surface to the geometric characteristics of the surface. In his dissertation [1], F. Noether studied the rolling of a ball on a surface of revolution. P. Woronetz more generally studied the rolling problem of a surface on another surface. A general discussion on the mechanics of these problems and more generally on nonholonomic problems and related literature can be found in [2]. Our viewpoint however will be more geometrical than mechanical. Our remark is that one can explore the nature of a surface by rolling experiments with a small ball letting it roll on the surface and measuring the speed of its center. In fact we prove the following:

Proposition. A compact oriented surface of $E^{3}$ is a sphere if and only if, for a small ball rolling freely without sliding on the surface, the velocity of its center is constant in magnitude, for all initial conditions.

Before starting the proof let us discuss some general facts on mechanics.

1. The configuration space describing the motion of a rigid body in space is the cartesian product $R^{3} \times S O(3)$. $S O(3)$ denotes the set of $3 \times 3$ real orthogonal matrices of determinant 1 . A point $\left(r^{0}, K\right)$ from $R^{3} \times S O(3)$ gives the position in space of a fixed point $O^{\prime}$ of the rigid body and the position of an orthonormal frame $E_{1}, E_{2}, E_{3}$ rigidly attached to the body at the point $O^{\prime}$. The columns of $K$ are precisely the coordinates of the vectors $E_{1}, E_{2}, E_{3}$. We call $K$ the moving frame. Each point of the body $P$ is described by means of two sets of coordinates $\left(x^{\prime}, y^{\prime}, z^{\prime}\right)$ with respect to $E_{1}, E_{2}, E_{3}$ and $(x, y, z)$ with respect to the standard frame $e_{1}, e_{2}, e_{3}$ of $R^{3}$. If $r^{0}=\left(x^{0}, y^{0}, z^{0}\right)$ are the coordinates of $O^{\prime}$, then the relation between the two sets of coordinates is

$$
r=r^{0}+K r^{\prime} .
$$

$r^{\prime}$ does not change during the motion (rigid body), hence differentiating (1) gives

$$
\dot{r}=\dot{r}^{0}+\dot{K} r^{\prime}=\dot{r}^{0}+\dot{K} K^{-1}\left(r-r^{0}\right) .
$$

$\dot{K} K^{-1}$ is an antisymmetric matrix and defines through its nonzero elements a vector $\omega$ such that

$$
\left(\dot{K} K^{-1}\right) v=\omega \times v
$$

Received by the editors June 26, 1984 .

1980 Mathematics Subject Classification. Primary 53A05; Secondary 53A17.

(C)1986 American Mathematical Society $0002-9939 / 86 \$ 1.00+\$ .25$ per page 
for every $v$ from $R^{3}$. $\times$ denotes the usual cross product of $R^{3}$. $\omega$ is classically called the "angular speed vector" of the rigid body. $\left(\dot{r}^{0}, \omega\right)$ can be considered as a tangent vector of the configuration space.

2. The equations of motion of a rigid body are most easily obtained from the d'Alembert-Lagrange principle (with Lagrange multipliers $\lambda_{i}$ ) for which the rigid body takes the form

$$
\left(F-m \ddot{r}^{0}\right) \cdot v+\left(L-\frac{d}{d t}\left(\sum I_{i j} \Omega_{j} E_{i}\right)\right) \cdot w+\sum_{1}^{m} \lambda_{i} \eta_{i}(v, w)=0
$$

for all $(v, w)$ tangent vectors of the configuration space. $m$ denotes the total mass of the body, $F$ the total force applied on the body, $L$ the total torque, $I=\left(I_{i j}\right)$ the inertia tensor with respect to the moving frame, $\Omega=\left(\Omega_{1}, \Omega_{2}, \Omega_{3}\right)$ the coordinates of the angular speed vector $\omega$ with respect to the moving frame and $\eta_{1}, \ldots, \eta_{m}$ are linear forms on the configuration space which express the nonholonomic constraints. The point $O^{\prime}$ is supposed to coincide with the center of mass of the body.

3. In the special case where the rigid body is a ball rolling freely without sliding and without external forces and torque, the equations are simplified to

$$
\left(-m \ddot{r}^{0}\right) \cdot v+\left(-\frac{d}{d t} I \omega\right) \cdot w+\lambda \cdot(v-w \times a N)=0 .
$$

The constant $I$ expresses the inertia tensor as a multiple of the identity, $a$ is the radius of the ball and $r^{0}$ is the center of the ball. The constraint

$$
v-w \times a N=0
$$

expresses the fact that the contact point of the two surfaces has zero instant velocity during the motion. $\lambda=\left(\lambda_{1}, \lambda_{2}, \lambda_{3}\right)$ are the Lagrange multipliers.

4. Equation (5) holds for all tangent vectors $(v, w)$, hence it gives

$$
\begin{gathered}
m \ddot{r}^{0}=\lambda, \\
I \frac{d}{d t} \omega=\lambda \times a N, \\
\dot{r}^{0}=\omega \times a N .
\end{gathered}
$$

Now we observe that $r^{0}$ lies on the parallel surface at distance $a$ on the side of rolling, hence is again a compact surface if $a$ is small enough. Taking tangential $(\cdots)^{T}$ and vertical $(\cdots)^{\perp}$ components of the equations (7)-(9) with respect to this parallel surface we get

$$
\omega^{T}=\frac{1}{a} N \times \dot{r}^{0}
$$

According to (8) the normal component of $\dot{\omega}$ is zero, hence

$$
(\omega \cdot N)^{\cdot}=\omega \cdot \dot{N}=\omega \cdot A\left(\dot{r}^{0}\right),
$$

where $A$ is the second fundamental tensor of the parallel surface with respect to the normal $N$. For the tangential component of $\dot{\omega}$ we obtain

$$
\begin{aligned}
(\dot{\omega})^{T} & =\left(\left(\omega^{T}\right)^{\cdot}\right)^{T}+\left(\left(\omega^{\perp}\right)^{\cdot}\right)^{T} \\
& =\nabla_{\dot{r}^{0} \omega^{T}+((\omega \cdot N) N)^{\cdot T}} \\
& =\nabla_{\dot{r}^{0}} \omega^{T}+(\omega \cdot N) A\left(\dot{r}^{0}\right) .
\end{aligned}
$$


Introducing (10) into (11) we obtain

$$
(\dot{\omega})^{T}=\frac{1}{a} N \times \nabla_{\dot{r}^{0}} \dot{r}^{0}+(\omega \cdot N) A\left(r^{0}\right) .
$$

Here $\nabla$ denotes the covariant derivative of the surface. Also (7) gives

$$
m \nabla_{\dot{r}^{\circ}} \dot{r}^{0}=\lambda^{T}
$$

and $(8)$ gives

$$
\begin{gathered}
I \dot{\omega}=\lambda^{T} \times a N=m a \nabla_{\dot{r}^{0}} \dot{r}^{0} \times N, \\
I \dot{\omega}=\lambda^{T} \times a N=m a\left(\nabla_{\dot{r}^{0}} \dot{r}^{0} \times N\right), \quad I(\dot{\omega})^{T}=m a\left(\nabla_{\dot{r}^{0}} \dot{r}^{0} \times N\right) .
\end{gathered}
$$

Hence according to (12)

$$
\frac{I}{a} N \times \nabla_{\dot{r}^{0}} \dot{r}^{0}+I \omega \cdot N A\left(\dot{r}^{0}\right)=m a\left(\nabla_{\dot{r}^{0}} \dot{r}^{0} \times N\right),
$$

which implies

$$
\left(\frac{I}{a}+m a\right) N \times \nabla_{\dot{r}^{0}} \dot{r}^{0}=I(\omega \cdot N) A\left(\dot{r}^{0}\right) .
$$

5. The proof of the proposition follows from (14). For if we suppose that $\dot{r}^{0}$ is constant in magnitude, then

$$
\nabla_{\dot{r}^{0}} \dot{r}^{0}=\left|\dot{r}^{0}\right| k\left(N \times \dot{r}^{0}\right),
$$

where $k$ is the geodesic curvature of the curve $r^{0}(t)$. Hence (14) becomes

$$
I(\omega \cdot N) A\left(\dot{r}^{0}\right)=-\left(\frac{I}{a}+m a\right)\left|\dot{r}^{0}\right| k \dot{r}^{0} .
$$

Especially, (15) holds for all initial conditions at, say, $p=r^{0}(0)$ with $\dot{r}^{0}(0)=v$ and $\omega(0)=w$, such that $w \cdot N(p) \neq 0$. Take such a $w$ and vary $v$ on the tangent plane at $p$. Then (15) implies that every direction of the parallel surface is a principal direction of the surface; hence the surface consists entirely of umbilic points, hence it must be a sphere [3, p. 259]. Since the parallel surface is a sphere, the original surface must be too. Using (14) one can also easily prove that on a sphere the magnitude of $\dot{r}^{0}$ is constant.

\section{BIBLIOGRAPHY}

1. F. Noether, Ueber die rollende Bewegung einer Kugel auf Rotationsflächen, Dissertation, München, 1909.

2. J. I. Neimark and N. A. Fufaev, Dynamics of nonholonomic system 3 , Transl. Math. Monos., vol. 33, Amer. Math. Soc., Providence, R.I., 1972.

3. B. O'Neill, Elementary differential geometry, Academic Press, New York, 1966.

Department of Mathematics, University of Crete, Iraklion, Crete, Greece 